(c) American Dairy Science Association, 2005.

\title{
Methionine Availability in Plasma of Dairy Cows Supplemented with Methionine Hydroxy Analog Isopropyl Ester
}

\author{
B. Graulet, C. Richard, and J. C. Robert \\ Center of Evaluation and Research in Nutrition, ADISSEO France SAS, 03600 Commentry, France
}

\section{ABSTRACT}

Adequate Met supply is especially important in the dairy cow for milk protein synthesis. Because of insufficient Met contents in the most frequently used feedstuffs, Met becomes limiting in the diet of the dairy cow. To restore the amino acid balance of the diet and consequently to optimize lactation performance, Met must be supplied in a protected form because of its high degradability as a free amino acid by rumen microorganisms. A new chemical derivative of Met, the isopropyl ester of the 2-hydroxy-4-(methylthio)-butanoic acid (HMBi) was tested for its metabolic fate by following the evolution of plasma concentrations of its metabolites (2-hydroxy-4-(methylthio)-butanoic acid (HMB), Met, isopropyl alcohol, and acetone) after spot-dose supplementation (50 g Met equivalent) to 15 cows. Results indicated that HMBi would be quickly absorbed and hydrolyzed into HMB and isopropyl alcohol, and then converted to Met and acetone, respectively. In our experimental conditions, the Met availability for cows was estimated to be $48.34 \pm 2.05 \%$ using a calibration curve established by modeling the area under the curve response to increasing doses of Met supplied as Smartamine $\mathrm{M}$, whose bioavailability ( $80 \%$ ) is considered the reference value. Plasma kinetics and bioavailability of Met were compared between HMBi and Smartamine $\mathrm{M}$ in the same cows. Comparison of the kinetics suggests that HMBi would be absorbed through the rumen wall providing good protection against rumen microorganisms. It can thus be concluded that HMBi is a new source of Met for ruminants with an acceptable bioavailability.

(Key words: dairy cow, methionine, methionine hydroxy analog ester, bioavailability)

Abbreviation key: AUC = area under the curve, BPMC = basal plasma Met concentration, HMB = 2hydroxy 4-(methylthio)-butanoic acid, HMBi = isopro-

Received November 24, 2004.

Accepted June 14, 2005.

Corresponding author: Jean-Claude Robert; e-mail: jean-claude. robert@adisseo.com. pyl ester of 2-hydroxy 4-(methylthio)-butanoic acid, MetDi = digestible Met, SmM = Smartamine M.

\section{INTRODUCTION}

It is now well accepted that Met is one of the most limiting AA for milk protein synthesis in the dairy cow fed a variety of rations, as demonstrated by postruminal infusions of increasing amounts of this specific AA (Schwab et al., 1992; Pisulewski et al., 1996). By measuring the responses in terms of milk protein production and AA flows to the small intestine to graded doses of postruminal supply of Met, Pisulewski et al. (1996) assumed that Met must contribute about $2.5 \%$ of the AA found in the protein truly digested in the small intestine. These results confirmed the previous estimations made by Rulquin et al. (1993). More recently, the daily Met requirements for lactating cows were reviewed by the National Research Council and adjusted to $2.4 \%$ in metabolizable protein (NRC, 2001).

Among the possible strategies, the optimization of Met intakes by modulation of diet composition is difficult and remains unsuccessful because feed ingredients that are rich in Met (such as corn-based complements) do not lead to the ideal AA profile (NRC, 2001). Indeed, when Met requirements were met, other AA (such as Lys) deficiencies appear such as considered in the AA submodel of the Cornell Net Carbohydrate and Protein System and in the AA submodel developed in France by Rulquin (cited in NRC, 2001). Supplements of crystalline Met in the ration have been excluded because free Met is quickly and almost totally degraded by the microorganisms in the rumen (NRC, 2001). Consequently, technologies have been developed to supply rumen-protected forms of Met available for dairy cow metabolism. A first strategy of protection was based on physical protection of Met using a surface coating made of a fatty acid and $\mathrm{pH}$-sensitive polymer mixture, or fat, saturated fatty acids, and mineral matrices. However, handling restrictions for feed manufacturing in the field prompted the suppliers to investigate alternative solutions such as chemically modified forms of Met. Among them, DL-2-hydroxy-4-(methylthio)-butanoic acid (HMB) was already used successfully for Met supply to monogastric animals. However, in dairy cows, HMB 
supplementation used to have an effect on rumen fermentations that induced an increase in the fat yield but extremely rarely in the protein yield (Bhargava et al., 1977; Lundquist et al., 1985; Hansen et al., 1991) Thus, HMB did not seem to effectively replace absorbed Met for milk protein synthesis.

In the present paper, the kinetic evolution of plasma Met concentrations following supplementation with the isopropyl ester of HMB (HMBi), a new form of rumenprotected Met, was described and compared with that of Smartamine M (SmM; Adisseo France SAS, Antony, France), a physically rumen-protected product for which Met bioavailability in lactating dairy cows is considered a reference in the field (Schwab, 1995). The Met bioavailability in SmM was first estimated to be $80 \%$ from the determination of its rumen resistance and intestinal availability by nylon bag studies, as reported by Schwab (1995). Later, Met availability in SmM was estimated using different experimental approaches and was determined to be 75.0 to $97.1 \%($ SED $=11)$ according to proportions of grass and corn silage in the diets estimated by digestibility tests (Robert and Williams, 1997) or $75 \pm 11 \%$ in a blood test in which the calibration curve was linear and established by steadystate levels of plasma Met concentrations after duodenal infusions of 0 to $30 \mathrm{~g}$ of Met in lactating cows (Rulquin and Kowalczyk, 2003). The aims of the present study were to determine the Met bioavailability of HMBi by using a relationship between the area under the curve (AUC) of plasma Met concentrations observed in response to increasing rumen spot-doses of Met (0 to $40 \mathrm{~g}$ ) supplied to dry, nongestating, mature cows as $\mathrm{SmM}$ used as a reference ( $80 \%$ of bioavailability).

\section{MATERIALS AND METHODS}

\section{Animals and Diets}

Nineteen mature, nongestating, dry Holstein cows (mean BW $=824 \pm 18 \mathrm{~kg}$ ) were used in this study. All cows had been surgically equipped with permanent ruminal cannulas (106 mm diameter; INRA, Theix, France) before the beginning of the current experiment. Cows were housed in individual stalls with natural temperature and lighting. The study was conducted in accordance with the European recommendations for the use of experimental vertebrate animals including animal welfare and appropriate conditions (Guidelines 86/ 609 of the European Union, November 24, 1986).

Due to their mature, dry, nongestating conditions, the cows were limit-fed and received individually twice a day a ration composed of permanent grassland hay and concentrate, the proportions and chemical composi-
Table 1. Ingredients and chemical composition of the basal diet. ${ }^{1}$

\begin{tabular}{lc}
\hline Ingredients $^{1}$ & $\%, \mathrm{DM}$ basis \\
\hline Hay & 75.6 \\
Barley grain & 9.9 \\
Dehydrated beet pulp & 9.0 \\
Soybean meal & 3.6 \\
Urea & 0.5 \\
Molasses & 1.2 \\
Chemical composition & \\
DM & 88.96 \\
Mineral matter & 6.86 \\
Crude fiber & 28.42 \\
Starch & 5.67 \\
CP & 12.87 \\
Fat & 1.38 \\
\hline
\end{tabular}

${ }^{1}$ Animals had free access to water and to a salt stone supplemented with micronutrients.

tion of which are presented in Table 1 . The concentrate was distributed at 0800 and $1545 \mathrm{~h}$, and the hay at 0800 and $1600 \mathrm{~h}$ in 2 equivalent meals. The average amounts of feed distributed were $2.05 \pm 0.03$ and 6.42 $\pm 0.10 \mathrm{~kg} / \mathrm{d}$ on a DM basis for concentrate and hay, respectively. Cows had free access to water. A salt lick was freely available to supply minerals $(4000 \mathrm{mg} / \mathrm{kg}$ of $\mathrm{Mg}, 10,000 \mathrm{mg} / \mathrm{kg}$ of $\mathrm{Zn}, 8250 \mathrm{mg} / \mathrm{kg}$ of $\mathrm{Mn}, 1500 \mathrm{mg} /$ $\mathrm{kg}$ of $\mathrm{Cu}, 120 \mathrm{mg} / \mathrm{kg}$ of Mo, $100 \mathrm{mg} / \mathrm{kg}$ of I, $100 \mathrm{mg} / \mathrm{kg}$ of Co, $15 \mathrm{mg} / \mathrm{kg}$ of Se; Sodioligo, Compagnie des Salins du Midi et des Salines de l'Est, Montpellier, France).

\section{Relationship Between Digestible Met and Plasma Met Concentrations}

Four cows were used in a $4 \times 4$ Latin square design composed of 4 successive 1 -wk periods and 4 treatments corresponding to the level of the Met supplementation given as a unique dose on the day of the experiment $(8.9,22.3,35.7$, and $49.1 \mathrm{~g})$. Methionine was supplied in the form of SmM, a source of Met physically protected by a $\mathrm{pH}$-sensitive coating, which is considered to have a Met bioavailability of $80 \%$ calculated from the determination of its rumen resistance $(90 \%)$ and its intestinal availability (90\%) by nylon bag studies (Schwab, 1995). Consequently, the doses of digestible Met (MetDi) added intraruminally directly in the liquid phase via the cannula, as a spot dose to follow the kinetics of plasma Met concentration, were 7.12, 17.84, 28.56 and $39.28 \mathrm{~g}$.

\section{Plasma Kinetics of Metabolites Following HMBi or SmM Supplementation}

The HMBi (MetaSmart; Adisseo France SAS) is a chemical derivative of HMB. Plasma concentrations of isopropyl alcohol, acetone, HMB, and Met were determined repeatedly on 15 dry, mature, nongestating Hol- 
stein cows following HMBi supplementation (50 g Met equivalent). Moreover, a direct comparison between HMBi and SmM was made on 7 of these cows according to a crossover design to compare their plasma Met evolution after supplementation with the 2 different products (50 g Met equivalent) and to validate the results of Met bioavailability calculated using the calibration curve.

\section{Item Supply}

The supplements (HMBi and SmM) were deposited directly through the cannula into the rumen at the level of the liquid phase just under the fibrous material. The HMBi was distributed at the time of the morning meal on the day of the experiment (d 1) and SmM was given at the time of the evening meal on the day before the experiment (d 0). Preliminary studies conducted in our laboratory (unpublished data) indicated that plasma Met response to SmM supplementation was preceded by a 10-h lag resulting from the retention of SmM in the rumen followed by progressive destruction of its coating matrix in the strongly acidic environment of the abomasum.

\section{Blood Sampling and Chemical Analysis}

For all 19 cows, blood samplings began on the day before the Met supplementation (d 0) at 0900, 1100, and $1500 \mathrm{~h}$. Then, on the day of supplementation (d 1), blood was collected regularly once every 1 or $2 \mathrm{~h}$ for the first $14 \mathrm{~h}$ postsupplementation. Finally, blood samples were obtained on the days after supplementation (d 2 and 3 ) at 0600, 0800, 1100, and $1500 \mathrm{~h}$. When SmM was used for Met supplementation, sampling was sustained until $72 \mathrm{~h}$ postsupplementation (d 4).

The blood samples were collected using an S-Monovette (Sarstedt Aktiengesellschaft \& Co., Nümbrecht, Germany) containing lithium chloride and heparin through the intermediary of a catheter (Cavafix Certo Splittocan 375 - B; Braun Medical Inc., Bethlehem, PA) positioned in the jugular vein. After centrifugation for $10 \mathrm{~min}$ at $1450 \times \mathrm{g}$, plasma was harvested then stored at $-20^{\circ} \mathrm{C}$ until subsequent metabolite analysis (HMB, Met, or solvents).

\section{Plasma HMB Assay}

The concentration of HMB was assayed in 2-mL plasma samples from the cows treated with HMBi. The macromolecular nitrogen was eliminated by treatment with an equal volume of acetonitrile followed by centrifugation for $10 \mathrm{~min}$ at $8100 \times g$ (room temperature). The supernatant was harvested and the pellet was rinsed with solution A (deionized water adjusted to $\mathrm{pH} 2.0$ with orthophosphoric acid $85 \%$ and acetonitrile in a $92: 8 \mathrm{ratio}, \mathrm{vol} / \mathrm{vol}$ ) and filtered using a $0.45-\mu \mathrm{m}$ filter (Millipore, Bedford, MA). The solution was pooled with the supernatant, the volume completed to $20 \mathrm{~mL}$ with solution $\mathrm{A}$, and the preparation was filtered using a cellulose disk AW03 (Millipore). The HMB was quantified in the samples by UV detection at $214 \mathrm{~nm}$ after separation on a Hibar prepacked RT-250-4 LichroSorb RP18 $(5 \mu \mathrm{m})$ column (Merck KgaA, Darmstadt, Germany) under isocratic conditions with an HPLC apparatus HP 1100 (Agilent Technologies, Palo Alto, CA). The mobile phase was solution A distributed at a flow rate of $0.8 \mathrm{~mL} / \mathrm{min}$. The injection volume of the samples was $20 \mu \mathrm{L}$. The linearity of HMB quantification was good between 2.0 and $134.2 \mu M$ of plasma. The recovery yield was 99.5 to $101.2 \%$ for concentrations between 13.4 and $134.2 \mu M$. The intra- and interassay variations were 0.1 and $<6 \%$, respectively. The concentration of HMB in the plasma samples was expressed in micromoles per liter after correction for standard variations and dilution values.

A previous experiment conducted in our laboratory had demonstrated that HMBi was undetectable (detection limit $=0.52 \mu M$ ) in the jugular plasma of the cows even though supplemented with $50 \mathrm{~g}$ of Met equivalent as HMBi (data not shown). Consequently, the HMBi assay in plasma was not performed in the present study.

\section{Plasma Met Assay}

Methionine analysis in plasma was performed after depletion of macromolecular nitrogen by treatment with $10 \%(\mathrm{vol} / \mathrm{wt}) 5$-sulfosalicylic acid $(3.15 M)$. After centrifugation for $10 \mathrm{~min}$ at $11,670 \times g$ (room temperature), the supernatant was collected. The pellet was washed several times with a lithium salt buffer $(\mathrm{pH}$ 2.2) containing $33.3 \mathrm{mM}$ trilithium citrate (Merck $\mathrm{KGaA}$ ), $35.2 \mathrm{~m} M$ citric acid, $0.5 \%$ thiodiethylene glycol (Sigma Chemical Co., St. Louis, MO), $0.28 \% \mathrm{HCl}$, and octanoic acid ( 2 drops for $2 \mathrm{~L}$ of buffer), and the supernatants were pooled. The final volume was adjusted to $5.0 \mathrm{~mL}$ with the lithium salt buffer and the preparation was filtered through a $0.2-\mu \mathrm{m}$ filter (Millipore) before storage at $4^{\circ} \mathrm{C}$ until assay. The Met concentration was determined in $50 \mu \mathrm{L}$ of sample by ion-exchange chromatography using a Beckman 6300 AA analyzer (Beckman Instruments, Palo Alto, CA) at $570 \mathrm{~nm}$ after postcolumn ninhydrin derivatization. Calibration was achieved with a $50 \mu M$ mixture (in $0.1 \mathrm{~N} \mathrm{HCl}$ ) of acid, neutral, and basic AA for calibrating AA analyzers (Sigma Chemical Co.). Finally, 1.25 mM of 2-amino-2-deoxy-Dgluconic acid (Sigma Chemical Co.) and S-(2-aminoethyl)-L-cysteine hydrochloride (Fluka, Buchs, Switzer- 
land) were used as internal standards in calibration solutions and samples. The lower limit of quantification of the Met concentration was established at $1.94 \mu M$ and the mean coefficient of variation was $0.92 \%$. Met concentration in the plasma samples was expressed in micromoles per liter after correction for calibration adjustment, internal standard variations, and dilution values.

\section{Plasma Isopropyl Alcohol and Acetone Assays}

Plasma concentrations of isopropyl alcohol and acetone were determined after HMBi supplementation in 4 cows with a GLC (type 8500; Perkin-Elmer, Norwalk, CT) equipped with a headspace injection system for specific determination of the volatile components of the samples. A $50 \mathrm{~m} \times 0.32 \mathrm{~mm}$ capillary column lined with a 1- $\mu \mathrm{m}$ dimethylpolysiloxane film (HP-1; Agilent Technologies) was used at a maximum temperature of $280^{\circ} \mathrm{C}$ to separate the volatile products, which were detected by flame ionization. Peak areas were calculated using the software of the chromatograph. Acetone and isopropyl alcohol concentrations in the samples were calculated from the difference in peak areas between isopropyl alcohol or acetone from the sample with or without adding an internal standard solution. Values were expressed in micromoles per liter of plasma.

\section{Data Analysis and Modeling}

Plasma kinetics of HMB and Met concentrations after HMBi supplementation were modeled using the nonlinear regression of the software Sigma Plot 6.00 (2000; SPSS Inc., Chicago, IL) and peak time after supplementation and height were determined.

The basal plasma Met concentration (BPMC) was determined for each cow as the mean of the Met values quantified at 0900,1100 , and $1500 \mathrm{~h}$ on $\mathrm{d} 0$, and at $0800 \mathrm{~h}$ on $\mathrm{d} 1$. The BPMC value was subtracted to concentrations determined for the respective cow after supplementation. The resulting values corresponded to the increases in plasma Met concentrations observed after supplementation and were used to draw the curves of Met increases related to the time after supplementation. The elemental trapezoidal AUC were calculated by multiplying the mean of the Met increases between 2 consecutive times of sampling and the duration of the interval between the samplings (Welling, 1986). The total AUC was the sum of the elemental AUC calculated all along the sampling kinetics.

The modeling of the relationship between MetDi doses and AUC quantification after supplementation with increasing doses of MetDi as SmM was performed using a nonlinear regression with the SigmaPlot 6.00 software (SPSS Inc.). The resulting relationship was used 1) to determine MetDi content from HMBi supplementation of the 15 cows, and 2) to compare MetDi values from HMBi and SmM on 7 cows of the 15 .

Finally, Met bioavailability in HMBi was calculated as the ratio between the digestible Met estimated from the calibration curve and the dose level of supplementation, and the result was expressed in percentage of the amount of Met or Met equivalent supplied by the product.

\section{Statistical Analyses}

Relationship between digestible Met and plasma Met concentrations. Data from Met kinetics were tested for normality, variance homogeneity, and compared by ANOVA using the StatView 5.0 software (SAS Institute, 1998) according to the following model:

$$
\mathrm{Y}_{\mathrm{ij} \kappa}=\mu+\alpha_{\mathrm{i}}+\beta_{\mathrm{j}}+\gamma_{\kappa}+\varepsilon_{\mathrm{ij} \kappa},
$$

where $\mathrm{Y}_{\mathrm{ij} \kappa}$ was the BPMC or the AUC calculated from Met concentrations, $\alpha_{\mathrm{i}}$ was the cow effect $(\mathrm{n}=4), \beta_{\mathrm{j}}$ was the period effect $(n=4), \gamma_{\kappa}$ was the dose level of MetDi supplemented as $\operatorname{SmM}(\mathrm{n}=4)$, and $\varepsilon_{\mathrm{ij} \kappa}$ was the residue.

Comparison of plasma Met kinetics and bioavailability from HMBi and SmM supplementations. Data from Met kinetics were tested for normality and variance homogeneity, and compared by ANOVA with the StatView 5.0 software (SAS Institute, 1998) according to the following model:

$$
\mathrm{Y}_{\mathrm{ij}}=\mu+\alpha_{\mathrm{i}}+\beta_{\mathrm{j}}+\varepsilon_{\mathrm{ij}},
$$

where $\mathrm{Y}_{\mathrm{ij}}$ was the BPMC, the Met peak time value or height after supplementation, the AUC calculated from Met concentrations, the MetDi in the spot dose or the bioavailability of the product, $\alpha_{\mathrm{i}}$ was the treatment effect (HMBi or SmM), $\beta_{\mathrm{j}}$ was the cow effect $(\mathrm{n}=7)$, and $\varepsilon_{\mathrm{ij}}$ was the residue.

\section{RESULTS AND DISCUSSION}

\section{Determination of the Relationship Between Digestible Met and Plasma Met Concentrations}

Among the in vivo approaches (in sacco digestibility, ruminal escape, blood test, or production performance in terms of lactation or growth), the blood test appeared the most convenient solution regarding accuracy, rapidity, ease of use, and possibility for encapsulated and chemically modified forms of methionine. Moreover, preliminary studies conducted in our laboratory (data not shown) had demonstrated that the shape of plasma 


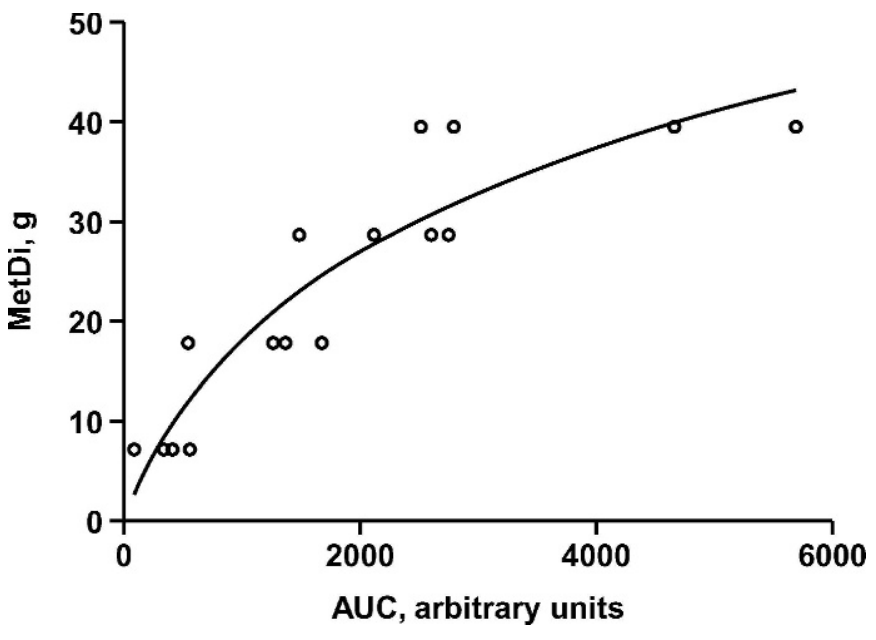

Figure 1. Modeling of the relationship between plasma Met response in term of AUC (area under the curve) and MetDi (digestible Met) values supplied as increasing doses of Smartamine M.

Met responses (slope and peak value) to spot-dose supplementations with rumen-protected products varied according to the protection technology, which could greatly interfere with Met bioavailability estimation. Consequently, it was estimated that the calculation of the AUC would better reflect the kinetics of plasma Met response to supplementations and would give a better estimate of Met bioavailability of the products. Thus, a calibration curve MetDi $=\mathrm{f}(\mathrm{AUC})$ was established, as described below.

Plasma Met concentrations were determined repeatedly for $72 \mathrm{~h}$ after spot-dose administration of increasing doses of SmM to 4 cows in a Latin square design. Kinetic evolutions were drawn and concentrations were used to calculate AUC values for each cow and each dose tested, after subtraction of the BPMC values. The cow effect was significant $(P=0.0245)$, but not the period or the treatment effects for the BPMC values. It suggested that each cow would have specific plasma Met status, at least before supplementation with additional Met. After supplementation with SmM, AUC values tended to depend on the cow $(P=0.0774)$ but as expected, the main effect was the treatment $(P=0.0009$; Figure 1).

Consequently, the modeling of the relationship MetDi $=f(A U C)$ was achieved by adding the origin intercept to the data list because without supplementation, the AUC value remains null. The model which best fit the data derived from the monomolecular model: MetDi $=18.1943 \times \ln (1+$ AUC/520.1822 $)$ with $\mathrm{R}^{2}$ adjusted $=0.833$ and standard error of estimate $=5.050$. The corresponding curve is represented in the Figure 1.

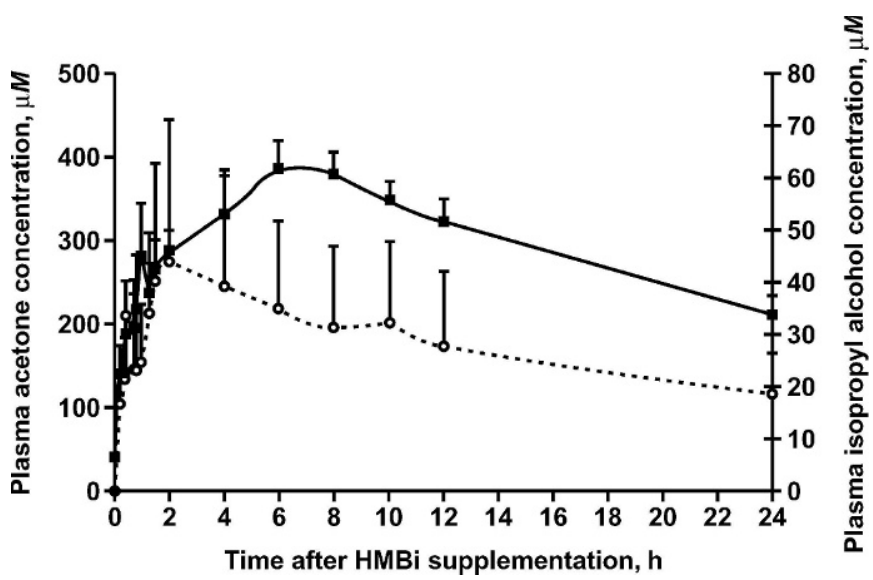

Figure 2. Kinetic variations of isopropyl alcohol $(O)$ and acetone (घ) concentrations in the plasma of 4 cows during the $24 \mathrm{~h}$ following intraruminal supplementation with a spot dose (50-g Met equivalent) of HMBi [2-hydroxy 4-(methylthio)-butanoic acid isopropyl ester].

\section{Evolution of Plasma Concentrations of Isopropyl Alcohol, Acetone, HMB, and Met Following HMBi Supplementation}

Isopropyl alcohol and acetone concentrations in plasma were determined in 4 of 15 cows during the 24 $\mathrm{h}$ following HMBi supplementation in the rumen with particular attention to the first $8 \mathrm{~h}$. As previously reported in the literature (Thin and Robertson, 1953), isopropyl alcohol was undetectable in peripheral plasma of control cows (Figure 2). Its plasma concentration quickly increased in the $2 \mathrm{~h}$ following HMBi supplementation to reach an average maximal value of 44.1 $\pm 27.5 \mu M$. This level was largely lower than the 1.4 $\mathrm{m} M$ reported for ketotic cows (Bruce and Lopez, 2000). Its plasma clearance by the liver was a natural physiological process. It was slow $\left(\mathrm{t}_{1 / 2}=19.8 \mathrm{~h}\right)$ but regular during the subsequent hours, at least until the end of the first $24 \mathrm{~h}$ postsupplementation.

The mean plasma acetone concentration was $41.7 \pm$ $3.1 \mu M$ before supplementation and it increased above the basal level as quickly as isopropyl alcohol concentration after the HMBi supplementation (Figure 2). However, the increase lasted almost $6 \mathrm{~h}$ and the concentration reached a peak value of $385.7 \pm 35.3 \mu M$. These concentrations were around 10-fold lower than values reported for ketotic dairy cows (Bruce and Lopez, 2000). Then, the concentration decreased linearly with a halflife of $22.6 \mathrm{~h}$. Based on the half-life and peak height values, the basal values of isopropyl alcohol and acetone have been estimated to be restored 40 and $46 \mathrm{~h}$ after supplementation, respectively.

Isopropyl alcohol and acetone metabolisms are tightly dependent, especially in ruminants. Indeed, iso- 


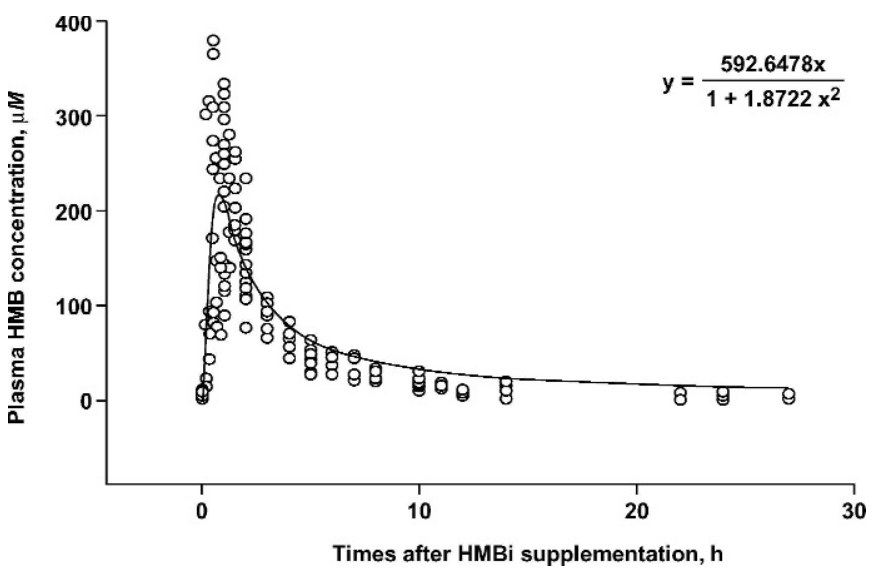

Figure 3. Modeling of the kinetic variations of HMB [2-hydroxy 4-(methylthio)-butanoic acid] concentrations in the plasma of 15 cows during the $27 \mathrm{~h}$ following intraruminal supplementation with a spot dose (50-g Met equivalent) of HMBi [2-hydroxy 4-(methylthio)-butanoic acid isopropyl ester].

propanol that could be easily absorbed through the stomach or the intestine wall would reach the liver to be oxidized by the hepatic enzymes of the alcohol dehydrogenase family (E.C. 1.2.1.3). The resulting acetone would be either oxidized into carbon dioxide after entering into the tricarboxylic acid cycle, or secreted back into the blood stream. In lactating dairy cows, acetone could also be used for hepatic neoglucogenesis or direct secretion into milk. Furthermore, plasma acetone could also return to the rumen (via direct transfer from blood capillaries of the rumen wall or via secretion into saliva) to be reduced back into isopropyl alcohol by the rumen microorganisms. In this way, it would constitute a metabolic cycle favoring the transfer of 2 reducing hydrogens from the rumen to the liver (Bruce and Lopez, 2000). In the present study, circulating isopropyl alcohol was likely resulting from HMBi hydrolysis and the rather high isopropanol and acetone halflives might be due to subsequent acetone-isopropyl alcohol recycling by rumen microorganisms. Moreover, the acetone concentration was nearly 10 -fold higher than the isopropyl alcohol concentration, suggesting that the liver process for oxidation of the alcohol into ketone is highly efficient and rapid, in agreement with its putative role in detoxification (Bruce and Lopez, 2000).

Plasma HMB concentrations after HMBi supplementation were determined on 15 cows for $27 \mathrm{~h}$ and used to model the response (Figure 3 ). The mean basal HMB concentration in plasma was very low $(3.26 \pm 1.00 \mu M$, $\mathrm{n}=15$; Table 2) and close to the limit of quantification $(2.3 \mu M)$ of the assay. However, HMB would not probably be a physiological plasma metabolite because Met catabolism by rumen microorganisms would produce preferentially the desulfurized (2-aminobutyric acid) and the oxidized forms (Met sulfoxide) rather than the deaminated form (HMB) of Met (Or-Rashid et al., 2001).

The plasma HMB kinetics (Figure 3) fit best with a standard rational model described by the relation $y=$ $a x /\left(1+b x^{2}\right)$ where $a=592.6478$ and $b=1.8722\left(\mathrm{R}^{2}=\right.$ 0.7019 , SEE (standard error of estimate $)=51.6632, P$ $<0.001$ ). The model seemed to indicate that the kinetics of HMB concentration in plasma comprised 2 phases. In the first $5 \mathrm{~h}$, plasma HMB concentrations varied very rapidly and the kinetics presented a peak shape. It could be seen that the HMB appearance following HMBi supplementation in the rumen was easily observable in the peripheral blood as soon as $10 \mathrm{~min}$ after supplementation in the rumen. Moreover, according to the model, the maximal HMB concentration (216.57 $\mu M$ ) was reached in the first hour following supplementation. On the other hand, HMBi is always undetectable in the plasma from peripheral blood both before and after supplementation in the rumen (unpublished data). Consequently, the increase in plasma HMB concentration after HMBi supplementation would result from HMBi absorption and then hydrolysis into HMB. Moreover, it is clear that the mechanism of HMBi transfer from the rumen juice to the peripheral blood, during which its hydrolysis into HMB occurs, is very rapid and efficient. However, the present knowledge does not allow determination of whether the hydrolysis process takes place in the lumen of the digestive tract (i.e., before absorption as HMB), or in the digestive wall or in the liver of the cow (after HMBi absorption).

Nevertheless, the lack of a lag time between HMBi supplementation and HMB peak development in the peripheral blood clearly suggests that HMBi absorption does not occur in the intestine-the mean retention time of the liquid phase in the rumen was estimated to be $8.7 \mathrm{~h}$ in dairy cows (Mambrini and Peyraud, 1997) and $10 \mathrm{~h}$ in grazing bovines (Lechner-Doll et al., 1991). Rather, it would indicate that HMBi was absorbed directly through the rumen wall, at least during the first $5 \mathrm{~h}$ following HMBi supplementation. Evidence for AA (or some of their chemical derivatives) absorption through the rumen wall has been already observed in vivo in multicatheterized sheep (Rémond et al., 2000), in empty-and-washed rumen (Veresegyhazy et al., 2001), or ex vivo by using parabiotic chambers (McCollum et al., 2000). Moreover, HMBi is a Met analog esterified with an isopropyl function that could change its lipophilic properties allowing its passage through biological membranes. Indeed, preliminary studies in multicatheterized ruminants or using the empty-andwashed rumen approach would confirm the efficient ruminal absorption of HMBi (Graulet et al., 2004; Noziere et al., 2004). Finally, a recent comparison of the 
Table 2. Characteristics of concentrations of plasma HMB [2-hydroxy 4-(methylthio)-butanoic acid] and Met evolution following HMBi [2-hydroxy 4-(methylthio)-butanoic acid isopropyl ester] supplementation and Met bioavailability of HMBi according to the method of the area under the curve. ${ }^{1}$

\begin{tabular}{lrr}
\hline Characteristic & \multicolumn{1}{c}{ HMB } & \multicolumn{1}{c}{ Met } \\
\hline Basal concentrations, $\mu M$ & $3.26 \pm 1.00$ & $20.29 \pm 0.74$ \\
Peak time after supplementation, h & $0: 59 \pm 0: 07$ & $4: 00 \pm 0: 23$ \\
Peak height after supplementation, $\mu M$ & $268.4 \pm 20.7$ & $145.9 \pm 5.34$ \\
Area under the curve, AU ${ }^{2}$ & & $1698 \pm 155$ \\
Digestible Met in the spot dose, g & & $24.20 \pm 1.02$ \\
Met bioavailability of HMBi, \% & & $48.34 \pm 2.05$ \\
\hline
\end{tabular}

${ }^{1}$ Values are means \pm SE for 15 cows supplemented by a spot dose of $50 \mathrm{~g} / \mathrm{d}$ Met equivalent.

${ }^{2} \mathrm{AU}=$ Arbitrary units.

rumen degradability of 3 Met sources (HMBi, HMB, and DL-Met) performed in lactating cows showed that only $2.3 \%$ of the HMBi dose of supplementation could bypass the rumen toward the omasum (Noftsger et al., 2005). Consequently, the significant increase in the milk protein concentration observed with the HMBi treatment in the latter study probably resulted from rumen absorption of HMBi more than a low passage rate to the intestine, in agreement with our present hypothesis. The end of the first phase took place around the fifth hour postsupplementation and was followed by a second phase, in which plasma HMB concentrations returned slowly to the basal values ( $24 \mathrm{~h}$ after supplementation). It is more difficult to explain the significance of this second phase in the 2-phase model because it might reflect the superposition of different processes of HMBi: absorption and hydrolysis at the rumen level, rumen bypass, and then intestinal absorption.

Plasma Met concentrations after HMBi supplementation were also determined on 15 cows for $48 \mathrm{~h}$ and used to model the response (Figure 4). The mean BPMC

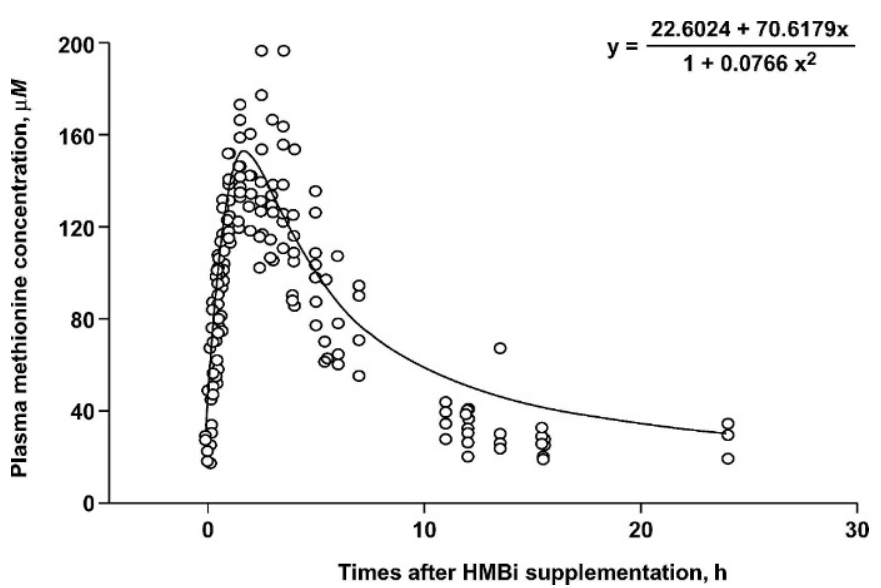

Figure 4. Modeling of the kinetic variations of Met concentrations in the plasma of 15 cows during the $48 \mathrm{~h}$ following intraruminal supplementation with a spot dose (50-g Met equivalent) of HMBi [2hydroxy 4-(methylthio)-butanoic acid isopropyl ester]. in plasma was $20.29 \pm 0.74 \mu M$ before supplementation, which corresponded to values observed in lactating cows (Pisulewski et al., 1996; Koenig et al., 2002). As for HMB concentrations, the kinetics of plasma Met concentrations (Figure 4 ) best fit with a standard rational model described by the relation $y=(a+b x) /(1+$ $c x^{2}$ ) where $a=22.6024, b=70.6179$, and $c=0.00766$ $\left(\mathrm{R}^{2}=0.744\right.$, standard error of estimate $=23.4872, P$ $<0.0001)$. Plasma Met concentration levels increased quickly during the first $3 \mathrm{~h}$ following HMBi supplementation (7-fold the basal values) until values exceeded $140 \mu M$ (Table 2). Then, Met concentrations decreased slowly to restore the basal values, as could be observed $48 \mathrm{~h}$ after supplementation. The comparison between HMB and Met kinetics seems to indicate that the HMB provided by HMBi supplementation was converted into Met by the cow tissues. Studies on monogastric species have shown that HMB would be first oxidized into 2keto-4-(methylthio)-butanoic acid before a transamination reaction process to produce Met (Dibner and Knight, 1984; Dibner and Ivey, 1992). The 2 enzymes acting in HMB conversion into 2-keto-4-(methylthio)butanoic acid were identified as L- $\alpha$-hydroxy acid oxidase (EC 1.1.3.15) and D-hydroxy acid dehydrogenase (EC 1.1.99.6) for the L- and D-HMB stereoisomers, respectively. Their activities have been shown to be the highest in the liver and kidneys but were also present in numerous other tissues in the chick (Dibner and Knight, 1984). Moreover, they were also described in the ruminal and omasal epithelium in the ovine (McCollum et al., 2000). Furthermore, Met synthesis from HMB was demonstrated in vivo to be especially active in the kidneys, liver, rumen, and the small intestinal parts of growing lambs (Wester et al., 2000). Consequently, it can be supposed that when supplemented intraruminally, HMBi was first absorbed by the rumen wall, then hydrolyzed into HMB and isopropyl alcohol, which were subsequently converted into Met by the cow tissues and acetone in the liver. 


\section{Met Bioavailability of $\mathrm{HMBi}$}

For each cow, the plasma Met concentrations corrected by the BPMC were used to determine an AUC value resulting from the HMBi supplementation (Table 2). The corresponding MetDi (i.e., metabolizable Met) was determined using the previous relationship. Thus, the mean digestible Met equivalent in the spot dose of HMBi was $24.20 \pm 1.02 \mathrm{~g}$, which corresponded to a mean Met bioavailability of $48.34 \pm 2.05 \%$ in HMBi (Table 2 ). To our knowledge, it is the first time a chemical derivative of hydroxy analog of Met was demonstrated to provide significant amounts of plasma Met to cows. Indeed, in previous studies, the resistance to fermentation by rumen microorganisms of several molecules, including methyl or ethyl esters of HMB, had been compared in vitro (Patterson and Kung, 1988). Those authors observed that methyl and ethyl esters of HMB were rapidly converted to $\mathrm{HMB}$, which was then degraded as observed in vitro (Windschitl and Stern, 1988) or in vivo (Langar et al., 1978; Jones et al., 1988). Then, they concluded that these molecules were not able to supply Met to cows (Patterson and Kung, 1988). More recently, another group reported HMB bypass values close to 40 to $50 \%$ observed from ruminal and duodenal digestibilities in dairy cows in early lactation (Koenig et al., 2002). The discrepancy with the previous results could be explained by the difference in the dose level or the mode of supplementation, by species differences, or by the physiological status of the animals. Moreover, HMB had a very moderate effect on plasma Met concentrations (Koenig et al., 2002) and did not increase milk protein content and yield (St-Pierre and Sylvester, 2005) indicating that HMB could not effectively meet the Met requirements of the dairy cow.

In the present study, around $50 \%$ of the HMBi would be protected from ruminal degradation by absorption through the rumen wall. The remaining 50\% would be hydrolyzed by the rumen microorganisms and the resulting HMB mostly degraded.

\section{Comparison of Met Plasma Kinetics and Bioavailability Between $\mathrm{HMBi}$ and SmM}

The comparison between HMBi and SmM was conducted in a crossover experimental design including 7 dry Holstein cows that received a direct spot supplementation into the rumen by cannula. Plasma Met kinetics were compared for $48 \mathrm{~h}$ after HMBi supplementation. Mean characteristics of the kinetics are presented in Table 3. The mean BPMC were not different between the 2 treatments and were similar to the previous measurements. The kinetics of plasma Met concentrations was very different between HMBi and SmM supplementations as illustrated by the peak description (Table
3). Indeed, as shown above, there was no lag-time after HMBi supplementation and the Met concentrations increased quickly to reach the peak value only $4 \mathrm{~h}$ after supplementation. The high values lasted only $4 \mathrm{~h}$, and then the plasma concentrations decreased slowly to restore the basal values $30 \mathrm{~h}$ after supplementation (data not shown). By contrast, the plasma Met concentrations were still at the basal values $10 \mathrm{~h}$ after $\mathrm{SmM}$ supplementation, then increased rapidly 5- to 6 -fold in the following $2 \mathrm{~h}$ to reach a 16-h lasting plateau (data not shown). The calculated mean peak time for SmM was $22.4 \mathrm{~h}$ and was significantly different from the HMBi value $(P=0.001)$. However, the peak heights were not different between the 2 products. Consequently, the AUC value for SmM was 1.87-fold higher than the HMBi value ( $P=0.0026$, Table 3$)$. The corresponding MetDi values were calculated with the calibration curve obtained above. Values for MetDi were significantly higher for SmM than for HMBi treatment $(\times 1.44, P=$ 0.0044). The mean Met bioavailability of SmM and HMBi estimated by this blood test method were 74.44 \pm 2.15 and $52.84 \pm 3.40 \%$, respectively. The value obtained for SmM was in agreement with the practical value (80\% reported by Schwab, 1995) and the previous estimations (close to $75 \%$ ) obtained by digestibility (Robert and Williams, 1997) and blood tests (Rulquin and Kowalczyk, 2003). This would indicate that the present calibration curve would be a useful tool to efficiently estimate Met bioavailability of different Metbased products. Finally, our results indicated that Met bioavailability from HMBi was as high as $71 \%$ of that of Met from SmM.

The overall comparison of the concentration kinetics between the 2 products tested allows us to propose an hypothesis about HMBi metabolism. Indeed, SmM is a small particle $(2 \mathrm{~mm})$ that is composed of a core of Met molecules coated with a $\mathrm{pH}$-sensitive matrix. The mean retention time of equivalent-sized particles in the digestive tract of grazing bovines has been estimated using marker-labeled particles to be $28 \mathrm{~h}$ in grazing animals (Lechner-Doll et al., 1991) and $31.9 \mathrm{~h}$ in lactating cows (Mambrini and Peyraud, 1997). Smartamine M migrates with the solid phase of small particles in the rumen juice and Met is released after passage in the abomasum where $\mathrm{pH}$ becomes strongly acidic. Finally, Met is absorbed at the jejuno-ileal level in the small intestine. The value of Met peak time after SmM supplementation was a plateau between 14 and $30 \mathrm{~h}$. Such observations thus agreed with the time required for SmM particles to migrate from rumen to small intestine and for Met to be released from the particle. On the other hand, the increases of HMB and Met concentrations after HMBi supplementation occurred too quickly after the supplementation to result from an intestinal 
Table 3. Characteristics of concentrations of plasma Met evolutions following HMBi [2-hydroxy 4-(methylthio)-butanoic acid isopropyl ester] or SmM (Smartamine M; Adisseo France SAS, Antony, France) supplementations and determination of Met bioavailability of these products according to the area under the curve method. ${ }^{1}$

\begin{tabular}{lrrrrr}
\hline & & & \multicolumn{2}{c}{$P$-value } \\
\cline { 3 - 6 } & \multicolumn{1}{c}{ HMBi } & SmM & Cow & Treatment \\
\hline Basal Met concentrations, $\mu M$ & $21.46 \pm 1.33$ & $21.41 \pm 1.31$ & 0.0709 & 0.9688 \\
Peak time after supplementation, $\mathrm{h}$ & $4: 00 \pm 0: 39$ & $22: 25 \pm 3: 30$ & 0.2743 & 0.0010 \\
Peak height after supplementation, $\mu M$ & $154.84 \pm 9.01$ & $178.91 \pm 12.84$ & 0.6779 & 0.2101 \\
Area under the curve, AU ${ }^{2}$ & $2005 \pm 282$ & $3994 \pm 290$ & 0.3539 & 0.0026 \\
Digestible Met in the spot dose, g & $26.42 \pm 1.70$ & $37.08 \pm 1.14$ & 0.7488 & 0.0044 \\
Met bioavailability of the product, $\%$ & $52.84 \pm 3.40$ & $74.44 \pm 2.15$ & 0.7716 & 0.0041 \\
\hline
\end{tabular}

${ }^{1}$ Values are means \pm SE for 7 cows supplemented by a spot dose of $50 \mathrm{~g} / \mathrm{d}$ Met equivalent.

${ }^{2} \mathrm{AU}=$ Arbitrary units.

absorption. Furthermore, isopropyl alcohol appeared also very quickly in plasma after HMBi supplementation, in agreement with the proposal that HMBi is absorbed through the rumen wall. The total absence of $\mathrm{HMBi}$ in the peripheral blood would suggest that $\mathrm{HMBi}$ is hydrolyzed before reaching the liver or in the liver. Consequently, several possible sites of hydrolysis could be proposed: just before absorption by the rumen wall, inside the rumen wall, or in the liver. It seems unlikely that HMBi hydrolysis would occur before absorption because, in the study of Koenig et al. (2002), the limited increase in plasma Met peak time after HMB supplementation to lactating dairy cows occurred $6 \mathrm{~h}$ after supplementation, indicating that HMB is not absorbed at the ruminal but at the intestinal level. However, further experiments are required to demonstrate effectively the sites of HMBi absorption and hydrolysis.

In the present experiment, Met bioavailability in HMBi was established to be $48 \%$ by a blood test method performed on 15 dry cows and using a calibration curve established with SmM. Moreover, the Met availability of HMBi corresponded to $71 \%$ of the value for SmM by direct comparison of the 2 products on 7 cows. The main evidence of these results is that HMBi provides substantial amounts of Met (as observed in plasma) to the cow, as this has been previously reported for SmM (Blum et al., 1999). Consequently, Met from HMBi would support milk protein synthesis in the mammary gland as observed with Met from SmM in lactating dairy cows. It can thus be concluded that HMBi is a very effective form of dietary Met supplementation for the lactating dairy cows.

\section{ACKNOWLEDGMENTS}

The authors thank Francis Pierre for analytical skills in plasma HMB and Met quantification, André Vergnol for plasma solvent quantifications, Bernard Bouza for his efficient technical help, and Laurent Delaval and Alain Bonichon for good care of the cows.

\section{REFERENCES}

Bhargava, P. V., D. E. Otterby, J. M. Murphy, and J. D. Donker. 1977. Methionine hydroxy analog in diets for lactating cows. J. Dairy Sci. 60:1594-1604.

Blum, J. W., R. M. Bruckmaier, and F. Jans. 1999. Rumen-protected methionine fed to dairy cows: Bioavailability and effects on plasma amino acid pattern and plasma metabolite and insulin concentrations. J. Dairy Sci. 82:1991-1998.

Bruce, M. L., and M. J. Lopez. 2000. Mixed ruminal microbes of cattle produce isopropanol in the presence of acetone but not 3D-hydroxybutyrate. J. Dairy Sci. 83:2580-2584.

Dibner, J. J., and F. J. Ivey. 1992. Capacity of the liver of the broiler chick for conversion of supplemental methionine activity to $\mathrm{L}$ methionine. Poult. Sci. 71:700-708.

Dibner, J. J., and C. D. Knight. 1984. Conversion of 2-hydroxy-4(methylthio)butanoic acid to L-methionine in the chick: A stereospecific pathway. J. Nutr. 114:1716-1723.

Graulet, B., C. Richard, and J. C. Robert. 2004. The isopropyl ester of methionine hydroxyl-analogue is absorbed through the rumen wall in the cow. J. Anim. Feed Sci. 13(Suppl. 1):269-272.

Hansen, W. P., D. E. Otterby, J. G. Linn, and J. D. Donker. 1991. Influence of forage type, ratio of forage to concentrate, and methionine hydroxy analog on performance of dairy cows. J. Dairy Sci. 74:1361-1369.

Koenig, K. M., L. M. Rode, C. D. Knight, and M. Vazquez-Añon. 2002. Rumen degradation and availability of various amounts of liquid methionine hydroxy analog in lactating dairy cows. J. Dairy Sci. 85:930-938.

Jones, B. A., O. E. Mohamed, R. W. Prange, and L. D. Satter. 1988 Degradation of methionine hydroxy analog in the rumen of lactating cows. J. Dairy Sci. 71:525-529.

Langar, P. N., P. J. Buttery, and D. Lewis. 1978. N-Stearoyl-D,Lmethionine, a protected methionine source for ruminants. J. Sci. Food Agric. 29:808-814.

Lechner-Doll, M., M. Kaske, and W. V. Engelhardt. 1991. Factors affecting the mean retention time of particles in the forestomach of ruminants and camelids. Pages 455-482 in Physiological Aspects of Digestion and Metabolism in Ruminants. T. Tsuda, Y. Sasaki, and R. Kawashima, ed. Academic Press, Inc., San Diego, CA.

Lundquist, R. G., D. E. Otterby, and J. G. Linn. 1985. Influence of three concentrations of DL-methionine or methionine hydroxy analog on milk yield and milk composition. J. Dairy Sci. 68:3350-3354.

Mambrini, M., and J. L. Peyraud. 1997. Retention time of feed particles and liquids in the stomachs and intestines of dairy cows. 
Direct measurement and calculations based on faecal collection. Reprod. Nutr. Dev. 37:427-442.

McCollum, M. Q., M. Vazquez-Añon, J. J. Dibner, and K. E. Webb, Jr. 2000. Absorption of 2-hydroxy-4-(methylthio)butanoic acid by isolated sheep ruminal and omasal epithelia. J. Anim. Sci. 78:1078-1083.

National Research Council. 2001. Pages 43-104 in Nutrient Requirements of Dairy Cattle. 7th rev. ed. Natl. Acad. Press, Washington, DC.

Noftsger, S., N. R. St-Pierre, and J. T. Sylvester. 2005. Determination of rumen degradability and ruminal effects of three sources of methionine in lactating cows. J. Dairy Sci. 88:223-237.

Noziere, P., C. Richard, B. Graulet, D. Durand, D. Rémond, and J. C. Robert. 2004. Investigation of the site of absorption and metabolism of HMBi and HMB in sheep. J. Dairy Sci. 87(Suppl. 1):220.

Or-Rashid, M. M., R. Onodera, S. Wadud, S. Oshiro, and T. Okada. 2001. Catabolism of methionine and threonine in vitro by mixed ruminal bacteria and protozoa. Amino Acids 21:383-391.

Patterson, J. A., and L. Kung, Jr. 1988. Metabolism of DL-methionine and methionine analogs by rumen microorganisms. J. Dairy Sci. 71:3292-3301.

Pisulewski, P. M., H. Rulquin, J. L. Peyraud, and R. Vérité. 1996. Lactational and systemic responses of dairy cows to post ruminal infusions of increasing amounts of methionine. J. Dairy Sci. 79:1781-1791.

Rémond, D., L. Bernard, and C. Poncet. 2000. Amino acid flux in ruminal and gastric veins of sheep: Effects of ruminal injections of free amino acids and carnosine. J. Anim. Sci. 78:158-166.

Robert, J. C., and P. Williams. 1997. Influence of forage type on the intestinal availability of methionine from a rumen protected form. J. Dairy Sci. 80(Suppl.1):248. (Abstr.)
Rulquin, H., and J. Kowalczyk. 2003. Development of a method for measuring lysine and methionine bioavailability in rumen-protected products for cattle. J. Anim. Feed Sci. 12:465-474.

Rulquin, H., P. M. Pisulewski, R. Vérité, and J. Guinard. 1993. Milk production and composition as a function of postruminal lysine and methionine supply: A nutrient-response approach. Livest. Prod. Sci. 37:69-90.

SAS Institute. 1998. StatView User's Guide. SAS Inst., Inc., Cary, NC. Schwab, C. G. 1995. Protected proteins and amino acids for ruminants. Pages 115-141 in Biotechnology in Animal Feeds and Animal Feeding. R. J. Wallace and A. Chesson, ed. V. C. H. Press, Weinheim, Germany.

Schwab, C. G., C. K. Bozak, N. L. Whitehouse, and M. M. A. Mesbah. 1992. Amino acid limitation and flow to the duodenum at 4 stages of lactation. 1. Sequence of lysine and methionine limitation. J. Dairy Sci. 75:3486-3502.

St-Pierre, N. R., and J. T. Sylvester. 2005. Effects of 2-hydroxy-4(methylthio)-butanoic acid (HMB) and its isopropyl ester on milk production and composition by Holstein cows. J. Dairy Sci. 88:2487-2497.

Thin, C., and A. Robertson. 1953. Biochemical aspects of ruminant ketosis. J. Comp. Pathol. 63:184-194.

Veresegyhazy, T., H. Febel, and A. Rimanoczy. 2001. Absorption of leucine, alanine and lysine from the rumen. Acta Vet. Hung. 49:81-86.

Welling, P. G. 1986. Pharmokinetics: Processes and Mathematics. American Chemical Society, Washington, DC.

Wester, T. J., M. Vazquez-Añon, D. Parker, J. Dibner, A. G. Calder, and G. E. Lobley. 2000. Synthesis of methionine (Met) from 2hydroxy-4-methylthio butanoic acid (HMB) in growing lambs. J. Dairy Sci. 83(Suppl.1):269. (Abstr.)

Windschitl, P. M., and M. D. Stern. 1988. Influence of methionine derivatives on effluent flow from continuous culture of ruminal bacteria. J. Anim. Sci. 66:2937-2947. 